\title{
Internal obturator muscle indirect injury in groin pain syndrome: a systematic review of the literature
}

\author{
Gian Nicola Bisciotti ${ }^{1}$ \\ Alessandro Corsini ${ }^{2}$ \\ Zarko Vuckovic ${ }^{1}$ \\ Emanuele Cena ${ }^{1}$ \\ Andrea Bisciotti ${ }^{3}$ \\ Alessandro Bisciotti ${ }^{3}$ \\ Andrea Belli ${ }^{2}$ \\ Alessandro Quaglia ${ }^{2,4}$ \\ Piero Volpi ${ }^{2,4}$ \\ 1 Qatar Orthopaedic and Sport Medicine Hospital, \\ Doha, Qatar \\ 2 Fc Internazionale, Milan, Italy \\ ${ }^{3}$ Centro Studi Kinemove Rehabilitation Centers, \\ Pontremoli (MS), Italy \\ ${ }^{4}$ Istituto Clinico Humanitas, Milan, Italy
}

Corresponding author:

Piero Volpi

Department Knee Orthopedic and Sports

Traumatology Unit

Humanitas Research Hospital

Via Manzoni 56

20089 Rozzano (MI), Italy

E-mail: piero.volpi@humanitas.it

\section{Summary}

Introduction: Traumatic groin pain syndrome can result from any acute trauma, but usually it is due to an indirect muscle injury (overstretching of the muscle fibers). The internal obturator muscle externally rotates the thigh and contributes to the stabilization of the hip joint. The indirect injury of this muscle may cause the onset of traumatic groin pain syndrome.

Purpose: To perform a systematic review of the literature concerning the indirect injury of internal obturator muscle in relationship to the onset of groin pain syndrome.

Conclusion: IO lesions are rare but important causes of traumatic groin pain syndrome, and despite their low incidence they must be considered during evaluation of patients with this pathology.

Study design: Systematic review.

Level of evidence: IV.
KEY WORDS: internal obturator, indirect lesion, groin pain syndrome, treatment.

\section{Introduction}

Groin Pain Syndrome (GPS) as proposed by the "Groin Pain Syndrome Italian Consensus Conference on Terminology, Clinical Evaluation and Imaging Assessment in Groin Pain in Athletics"1 can be defined as "any clinical symptom reported by the patient, located at the inguinal-pubic-adductor area, affecting sports activities and/or interfering with Activities of Daily Living and requiring medical attention".

GPS can also be divided into three main categories:

I. GPS due to functional overload, characterized by insidious and progressive onset, without an acute trauma, or a situation to which the onset of pain symptoms can be attributed with certainty

II. GPS of traumatic origin, in which the onset of pain was due to any acute trauma, and this hypothesis is supported by medical history, clinical examination and imaging

III. Long-standing GPS or chronic GPS, in which the cohort of symptoms reported by the patient continues for a long period (over 12 weeks) and is recalcitrant to any conservative therapy.

Traumatic GPS is usually due to acute muscletendon problems (indirect muscle-tendon injuries), and while the most affected muscles are rectus abdominis, adductors and iliopsoas muscle ${ }^{1}$, the internal obturator muscle $(\mathrm{IO})$ lesion is very rare ${ }^{2}$. IO is part of the so called "short lateral rotators" (i.e. piriformis, obturator internus, superior and inferior gemelli, obturator externus, and quadratus femoris) ${ }^{2}$. These muscles give an important contribution to the hip joint stability and are considered by some Authors as the "rotator cuff" of the hip ${ }^{3,4}$.

\section{Material and methods}

This systematic review was conducted in accordance with the PRISMA (Preferred Reporting Items for Systematic Reviews and Meta-Analysis) guidelines ${ }^{5}$. The protocol of this study was registered at the PROSPERO register (website: http://www.crd.york.ac.uk/ PROSPERO) for systematic reviews. 


\section{Aim of the current systematic review}

The aim of the current systematic review is to systematically underpin the role of 10 direct injury in GPS of traumatic origin, according to the Preferred Reporting Items for Systematic Reviews and Metaanalyses (PRISMA) guidelines ${ }^{6}$, as well as to the "Muscle, Ligament and Tendon Journal" guidelines?.

\section{Search strategy}

Prior to this systematic review the Cochrane Database of Systematic Reviews; MEDLINE and PEDro where searched for systematic reviews on GPS caused by 10 indirect injuries in order to make sure that similar systematic reviews are not already present in literature. After this verification two Authors (BGN and VP) independently searched the literature using a string of keywords such as "groin pain syndrome", "pubalgia" "Gilmore's groin", "hockey groin", "incipient hernia", "groin disruption", "inguinal disruption", "inguinal related groin pain ", "adductor-related groin pain", "iliopsoas-related groin pain", "pubic-related groin pain", "sportsman's hernia", "sportsman's groin", "sport hernia", "internal obturator", "tear", "lesion" and "injury" properly connected by Boolean operators. If appropriate, medical subject headings (MeSH) and wild-card options were used. Target journals were hand-searched, in order to increase the possibility to obtain all the relevant articles. The research was conducted on December $2^{\text {nd }} 2017$, there were no data restriction, no language limitation was applied and the so called "grey literature" (i.e. conference, abstracts, thesis and un- published reports) was not taken in consideration. The cross-reference from retained articles were screened to verify their possible relevance. All the double citation were removed. For each article, the relevant information was extracted and recorded on an ad hoc Excel spreadsheet. The details of search strategy are showed in Table I while the PRISMA flow diagram of the study search and selection procedure is showed in Figure 1.

\section{Data extraction, synthesis and analysis}

Using a standardized form two reviewers (GNB and $\mathrm{PV}$ ) extracted the following data from the eligible studies:

I. Study design

II. Participants

III. Study setting (sport type, level, gender, age)

IV. Injury etiology

V. Diagnosis

VI. Type of treatment (conservative, medical or surgical)

VII. Time loss injury

VIII. Outcome

Data was analyzed descriptively.

\section{Results}

After screening 134 articles 6 have been included and synthesized in the current systematic review. Characteristics of included studies are shown in Table II. All studies were checked for reporting on conflict of interest ${ }^{8}$.

Table I. Search strategy used in the study.

\begin{tabular}{ll}
\hline Search strategy items & Details \\
\hline Searched databases & PubMed/MEDLINE, Scopus, ISI, EXCERPTA MEDICA. \\
Searched string & $\begin{array}{l}\text { (groin pain syndrome OR pubalgia OR Gilmore's groin OR hockey groin OR incipient hernia } \\
\text { OR groin disruption OR inguinal disruption OR inguinal-related groin pain OR adductor- } \\
\text { related groin pain OR iliopsoas-related groin pain OR pubic-related groin pain OR } \\
\text { sportsman's hernia OR sportsman's groin OR sport hernia OR) AND (internal obturator) } \\
\text { AND (tear OR lesion OR injury) } \\
\text { P: Articles investigating GPS caused by IO muscle indirect injury at any level } \\
\text { I: conservative, medical or surgical intervention C: None, there are no comparison } \\
\text { parameters O: time loss injury associated by IO indirect injury causing GPS and its } \\
\text { outcome }\end{array}$ \\
& $\begin{array}{l}\text { P: Articles investigating IO overuse injuries or other causes not related to indirect injuries. I: } \\
\text { Articles not related to the conservative, medical or surgical intervention of IO indirect injury. } \\
\text { C: None, there are no comparison parameters O: Articles not focused on conservative, } \\
\text { medical or surgical intervention for IO indirect injury }\end{array}$
\end{tabular}

Time filter

None set

Language filter

None set

Target journals

All the peer-review and indexed journal are present in the considered data-base 


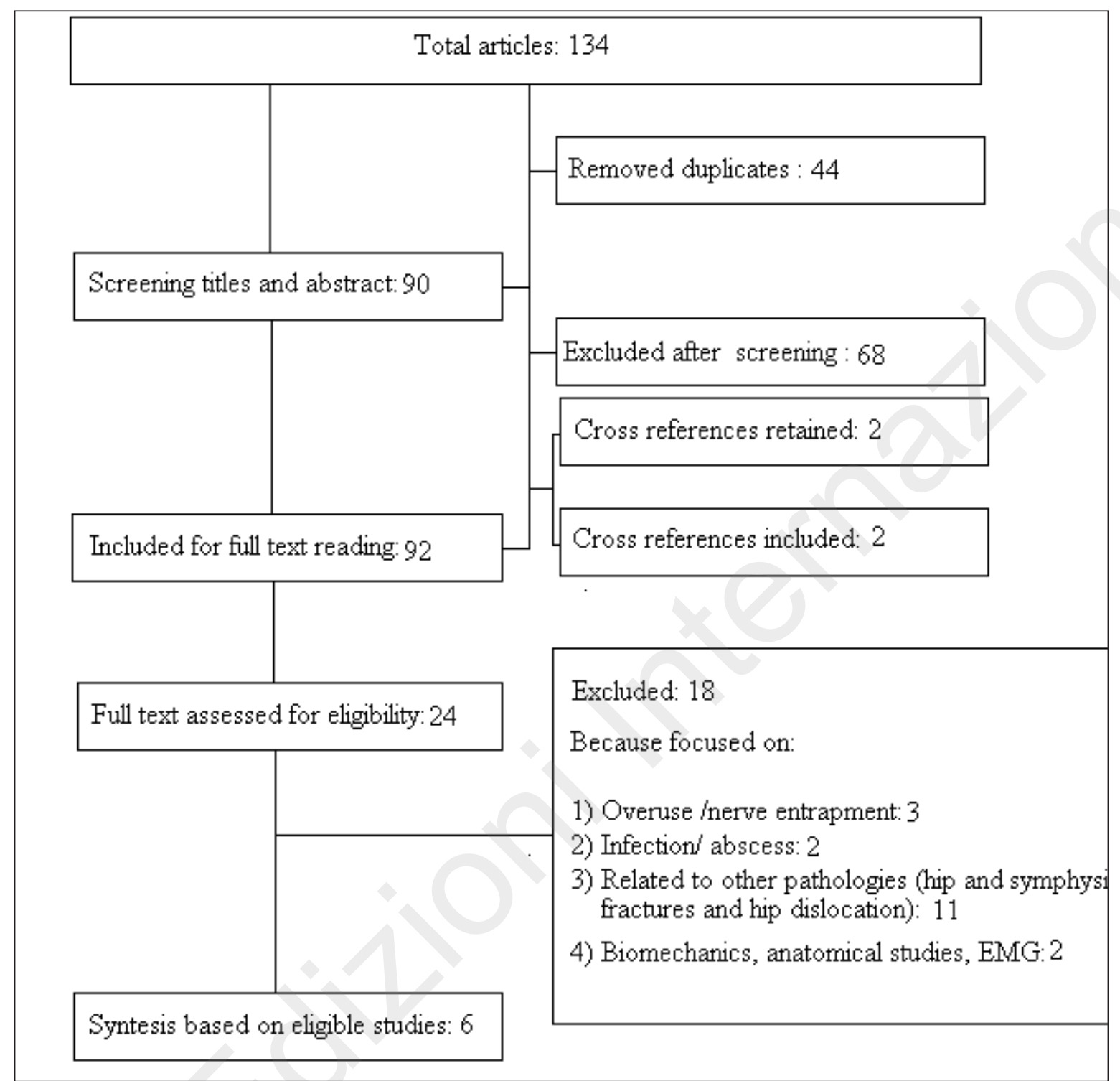

Figure 1. PRISMA flow diagram of the study search and selection procedure.

\section{Study design}

Five case reports ${ }^{9-13}$ (level of evidence IV) and only one retrospective observational study ${ }^{14}$ (level of evidence IV) were included in this systematic review, and the reason for such a small number of studies can be the relative rarity of $1 \mathrm{O}$ direct injuries.

\section{Study setting}

The studies setting take in consideration a population of young subjects (range 13-28 years) composed of both professional and amateur athletes. It is interesting to underline that sport activities of the considered athletes include strong and sudden movements of torsion and flexion of the upper body. Furthermore, excluding the study of Wong-On et al. ${ }^{14}$, which makes no difference between injury at 10 or external obturator level (EO), in 4 over 5 of the considered studies the injuries at 10 level occur in competition, and only one study ${ }^{9}$ does not specify whether the described injury occurred during training or in competition.

\section{Injury ethiology}

The IO injuries are in relationship with sudden movements of torsion and flexion of the upper body, like unstable change of direction ${ }^{9}$, kicking the ball in an unstable position $^{9}$, during a fall ${ }^{11}$, and during twisting ${ }^{12,13}$ or bending ${ }^{10}$ movements performed in competition.

\section{Diagnosis}

The diagnosis of $1 \mathrm{O}$ indirect injury was formulated in all cases by clinical and imaging assessment.

The clinical assessment was based on passive hip in- 


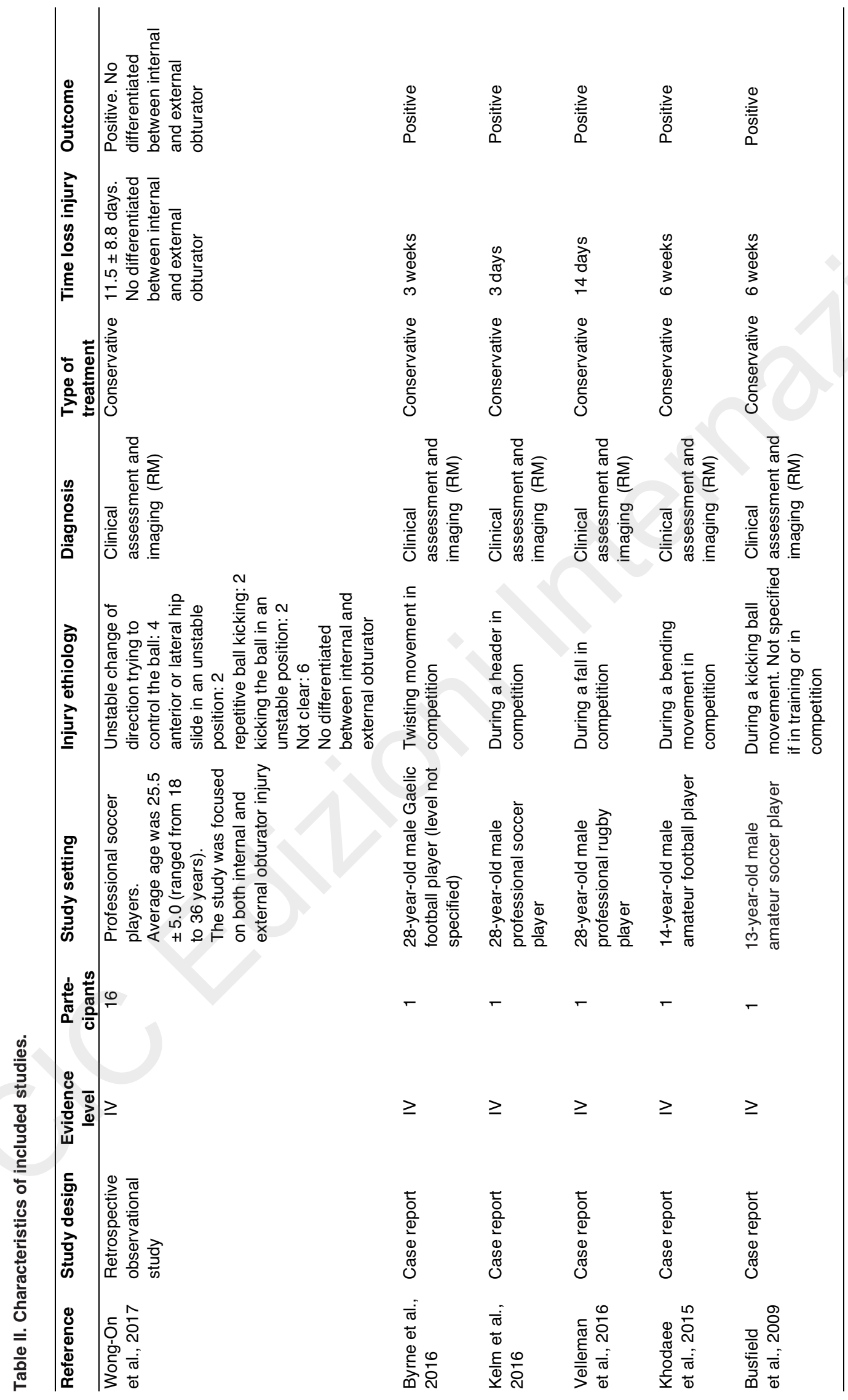


ternal and external rotation test. The tests are positive for injury if they cause pain during hip flexion and internal or external rotation passively or against resistance ${ }^{13,14}$. Usually the patient complains of pain at ischial tuberosity on deep pressure ${ }^{10,12}$, but sometimes there can be no pain on palpation ${ }^{11}$. There is usually no visible swelling, bruising, oedema or ecchymosis at inguinal, gluteal or posterior thigh area ${ }^{10,11}$. The patient's history is often positive for instability of the hip joint ${ }^{14}$.

All the considered studies based the imaging assessment on MRI scan. Several Authors considered the MRI essential to the location, classification, and evaluation of the injury size ${ }^{11,14}$. However, is important to underline that also the US examination shows a high sensitivity and specificity in 10 assessment ${ }^{15}$. Concerning MRI assessment some Authors suggest the use of standard high-field -1.5 or $3 T^{11}$. The hip MRI imaging was performed using PD, T2-weighted, FS, and/or STIR sequences ${ }^{13}$ in the axial, coronal, and sagittal planes ${ }^{14}$. The injury was classified in only two studies $^{13,14}$, and in both studies the type of used classification was accurately described. However, in the study of Wong-On et al. ${ }^{14}$ there was no differentiation between the lesions of $\mathrm{OI}$ and those of EO. In the others studies, there was only a description of the MRI signal changes ${ }^{9,12}$, or were used very vague and non-specific terms like "acute injury", "partial injury," "no complete disruption" 11 , "high grade strain"10.

\section{Treatment}

In all considered studies the rehabilitation program was based on both conservative and medical treatment. The conservative treatment utilized was: aquatic therapy ${ }^{10}$, cycling ${ }^{11}$, electrical stimulation ${ }^{10}$, gait training ${ }^{9}$, hot/cold modalities ${ }^{10}$, infra-red light therapy $^{10}$, iontophoresis ${ }^{10}$, manual lymphatic drainage ${ }^{12}$, training modification and restriction ${ }^{13}$, on line running ${ }^{11}$, rest ${ }^{11,13}$, strengthening and functional movement of the hip muscles ${ }^{9-12}$, swimming ${ }^{11}$ and ultrasound therapy ${ }^{10,12}$.

The medical treatment included nonsteroidal anti-inflammatories drugs ${ }^{11,13}$ and paracetamol ${ }^{10}$.

No study considered surgical treatment.

\section{Time loss injury}

Excluding the study of Wong-On et al. ${ }^{14}$ (in which there is no differentiation between injury at IO or EO level) the time loss injury was on average $24.40 \pm 17.30$ days (range 3-42 days).

\section{Outcome}

The outcome was in all the considered studies positive and all the subjects had a complete recovery and were able to return to full sport activity.

\section{Discussion}

IO is a flat triangular muscle and is part of the hip external-rotator muscle group. It originates from the inner surface of the obturator membrane and the surrounding bony margins of the obturator foramen. Its muscular bands pass then through the ischial foramen to become extra pelvic and to form a tendon origin that is oriented in antero-posterior direction. Finally $I O$ inserts at the medial surface of the great trochanter, in antero-posterior to the trochanteric pit. At the level of its distal insertion, the 10 blends with the tendon of the gemelli muscles (triceps coxae) forming a common tendon 2,16,17. This anatomical association with the gemelli tendon can explain the less frequent injuries of 10 in comparison to $\mathrm{EO}^{14}$. The $\mathrm{IO}$ externaly rotates the thigh by abducting it when the hip is flexed, and it also contributes to the stabilization of the hip joint. It is innervated by the obturator nerve (L5, S2) and its blood supply is provided by obturator artery. The maximum strength of 10 depends on the flexion degree of the hip joint and is maximal at the beginning of the oscillation phase during the run stride $^{18}$. In addition to the 10 , the other muscles working as hip external rotators are piriformis, external obturator, gemelli and quadratus femoris. These muscles are known under the collective name of "short lateral rotators"16. This muscle group performs a major hip-joint stabilizing action, stabilizing the femoral head within the acetabulum during hip movements ${ }^{2,16}$.

Despite the important work done by this muscle group, little is found in literature concerning the possible problems related to its injuries. The present study is, at our knowledge, the first systematic review present in literature focused on GPS caused by an indirect injury at IO level. Only six studies are present in literature focused on this topic, of which five are case reports ${ }^{9-13}$ (level of evidence IV) and one retrospective observational study ${ }^{14}$ (level of evidence IV). From the analysis of these studies we can deduct the following points.

\section{Injury ethiology}

There are essentially three main mechanisms causing traumatic 10 injury.

The first is an unstable pelvis position with a sudden change in body weight distribution ${ }^{14}$. The second is a sudden change of direction in condition of pelvic instability ${ }^{13,14}$. Finally, the third is represented by a sudden hip internal-rotation with hip and knee flexed at about $90^{\circ} 9$. The fact that re-injuries of the 10 are not described in the literature ${ }^{14}$ confirms the hypothesis that they are dependent on a well-defined biomechanical situation, such as those described above, and are independent of predisposing intrinsic factors such as excessive retraction or stiffness of the $1 \mathrm{O}^{14}$.

\section{Diagnosis}

The diagnosis of 10 indirect injury, like for the other muscles, is based on clinical and imaging assessment.

The most reliable clinical test for 10 injury is the 
passive internal rotation maneuver with and without muscle opposition by the patient ${ }^{12,14}$. The clinical presentation of $1 O$ indirect muscle injury is a GPS of traumatic origin framework $9,11,13,14$. The differential diagnosis to consider are pyriformis syndrome, femoroacetabular impingement, hip joint labral tear, rectus femoris tear, iliopsoas-related GPS, adductor-related GPS, inguinal-related GPS and pubic-related GPS ${ }^{14}$.

The MRI represents the imaging gold standard ${ }^{11,14}$. The suggested MRI protocol is utilization of a standard high-field (1.5 or $3 \mathrm{~T})^{11}$ performing PD, T2weighted, FS, and/or STIR sequences ${ }^{13}$ in the axial, coronal, and sagittal planes ${ }^{14}$. However, it possible also to consider US scan ${ }^{15}$.

\section{Treatment}

The treatment of 10 injuries is conservative and medical ${ }^{9-14}$, and does not differ from the treatment suggested for others indirect muscle injuries ${ }^{19}$.

\section{Time loss injury}

Time loss injury for a 10 indirect injury $(24.40 \pm 17.30$ days/range 3-42 days) is in line with the time loss injury that we can find in literature concerning other muscles involved in sport activity, like the biceps femoris $20 \pm 15$ days $^{20}$, the semitendinosus $18 \pm 11$ days $^{20}$, the semimembranosus $23 \pm 14$ days $^{20}$, the rectus femoris $18.00 \pm 4.24$ days $^{21}$ and GPS (24.3 days $)^{22}$. From epidemiological point of view it is interesting to note that Wong-On et al. ${ }^{14}$ in their observational cross-sectional study reporting injury data recorded in four seasons in the professional Spanish football league (La Liga), showed that there were 4 indirect IO injuries during the considered period.

\section{Outcomes}

IO injuries, usually have good prognosis with optimal treatment and a recovery time substantially overlapping that of other more frequently injured muscle groups ${ }^{19}$.

\section{Conclusions}

IO lesions represent the rarest lesions among hip external rotators, muscle group which shows a very low lesion incidence ${ }^{12,14}$. Etiology of 10 injuries is related to well-defined biomechanical situations and seems independent from intrinsic factors ${ }^{14}$ The clinical diagnosis of IO injury presents some objective difficulties. Indeed, IO-related lesion usually causes painful symptoms at the anterior hip-joint level. For this reason 10 injuries are in differential diagnosis with many other hip and groin clinical conditions ${ }^{14,23,24}$. Finally, it is important to remember that even though in general hip external-rotator muscle lesions (IO in particular) represent a rare occurrence ${ }^{12,14}$ they must be considered in clinical evaluation of a traumatic GPS framework. In any case, IO injuries show a good prognosis and relatively short recovery times.

\section{References}

1. Bisciotti GN, Volpi P, Zini R, et al. Groin Pain Syndrome Italian Consensus Conference on terminology, clinical evaluation and imaging assessment in groin pain in athlete. BMJ Open Sport Exerc Med. 2016;2:e000142. Doi:10.1136/ bmjsem2016-000142.

2. Yoo S, Dedova I, Pather N. An appraisal of the short lateral rotators of the hip joint. Clin Anat. 2015;28(6):800-812.

3. Lockhart RD, Hamilton GF, Fyfe FW. Anatomy of the Human Body. 2nd Ed. Faber \& Faber Ltd (Ed). London. 1965.

4. Pine J, Binns M, Wright $P$, Soames R. Piriformis and obturator internus morphology: A cadaveric study. Clin Anat. 2011;24: 70-76.

5. Moher D, Shamseer L, Clarke M, Ghersi D, Liberati A, Petticrew M, Shekelle P, Stewart LA. PRISMA-P Group. Preferred reporting items for systematic review and meta-analysis protocols (PRISMA-P) 2015 statement. Syst Rev. 2015 Jan 1;4:1.

6. McLeroy KR, Northridge ME, Balcazar H, Greenberg MR, Landers SJ. Reporting guidelines and the American Journal of Public Health's adoption of Preferred Reporting Items for Systematic reviews and Meta-Analyses. Am J Public Health. 2012 May;102(5):780-784. Doi: 10.2105/AJPH.2011.300630. Epub 2012 Mar 15.

7. Padulo J, Oliva F, Frizziero A, Maffulli N. Muscles, Ligaments and Tendons Journal - Basic principles and recommendations in clinical and field science research: 2016 update. MLTJ. 2016;6(1):1-5.

8. Shea BJ, Hamel C, Wells GA, Bouter LM, Kristjansson E, Grimshaw J, Henry DA, Boers M. AMSTAR is a reliable and valid measurement tool to assess the methodological quality of systematic reviews. J Clin Epidemiol. 2009 Oct;62(10):10131020.

9. Busfield BT, Romero DM. Obturator internus strain in the hip of an adolescent athlete. American Journal of Orthopedics (Belle Mead, NJ). 2009;38(11):588-589.

10. Khodaee M, Jones D, Spittler J. Obturator internus and obturator externus strain in a high schoolquarterback. Asian $\mathrm{J}$ Sports Med. 2015;6(3)23481.

11. Velleman MD, Jansen Van Rensburg A, Janse Van Rensburg $\mathrm{DC}$, Strauss $\mathrm{O}$. Acute obturator internus muscle strain in a rugby player: a case report. J Sports Med Phys Fitness. 2015 Dec;55(12):1544-1546.

12. Kelm J, Ludwig O, Schneider G, Hopp S. Injury of the Obturator Internus Muscle- a Rare Differential Diagnosis in a Soccer Player. Sportverletz Sportschaden. 2016 Mar;30(1):50-53.

13. Byrne C, Alkhayat A, O'Neill P, Eustace S, Kavanagh E. Obturator internus muscle strains Radiol Case Rep. 2017 Mar;12 (1):130-132. Published online 2016 Dec 16. Doi: 10.1016/j. radcr.2016.10.020.

14. Wong-On M, Turmo-Garuz A, Arriaza R, Gonzalez de Suso JM, Til-Perez L, Yanguas-Leite X, Diaz-Cueli D, Gasol-Santa $X$. Injuries of the obturator muscles in professional soccer players. Knee Surg Sports Traumatol Arthrosc. 2017 Feb 10. Doi: 10.1007/s00167-017-4453-6. [Epub ahead of print].

15. Wu R, Hu B, Ma F, Kuang S-L, Huang Y, Li Q, Yao M-H. A comparative study of the sonographic appearance and anatomy of the obturator internus in normal males. Minim Invasive Ther Allied Technol. 2012;21:271-275.

16. Gudena R, Alzahrani A, Railton P, Powell J, Ganz R. The anatomy and function of the obturator externus. Hip Int. 2015;25(5):424-427.

17. Meknas K, Kartus J, Letto J, Christensen A, Johansen O. Surgical release of the internal obturator tendon for the treatment of retro-trochanteric pain syndrome: a prospective randomized study, with long-term follow-up. Knee Surg Sports Traumatol Arthrosc. 2009;17:1249-1256. 
18. Solomon LB, Lee YC, Callary SA, Beck M, Howie DW. Anatomy of piriformis, obturator internus and obturator externus: implications for the posterior surgical approach to the hip. J Bone Joint Surg Br. 2010;92(9):1317-1324.

19. Bisciotti GN, Volpi P, Amato M, Alberti GP, Allegra F, Aprato A, Artina $\mathrm{M}$, Auci $\mathrm{A}$, et al. Italian consensus conference on guidelines for conservative treatment on lower limb Muscle injuries in athlete. Part II: clinical assessment and conservative treatment. BJSM. 2017, submitted.

20. Ekstrand J, Lee JC, Healy JC. MRI findings and return to play in football: a prospective analysis of 255 hamstring injuries in the UEFA Elite Club Injury Study. Br J Sports Med. 2016 Jun;50(12):738-743.

21. Ekstrand J. UEFA Elite Club Injury Study Report 2015/16. UEFA Medical Committee, 2016.

22. Mosler AB, Weir A, Eirale C, Farooq A, Thorborg K, Whiteley RJ, Hölmich P. Crossley KM Epidemiology of time loss groin injuries in a men's professional football league: a 2-year prospective study of 17 clubs and 606 players. Br J Sports Med. 2017 Jun 30. pii: bjsports-2016-097277. Doi: 10.1136/bjsports-2016-097277. [Epub ahead of print].

23. Griffin DR, Dickenson EJ, O'Donnell, Agricola R, Awan T, Beck M, Clohisy JC, Dijkstra HP, Falvey E, Gimpel M, Hinman RS, Hölmich P, Kassarjian A, Martin HD, Martin R, Mather RC, Philippon MJ, Reiman MP, Takla A, Thorborg K, Walker S, Weir A, Bennell KL. The Warwick Agreement on femoroacetabular impingement syndrome (FAl syndrome): an international consensus statement. Br J Sports Med. 2016;50:11691176.

24. Weir A, Brukner P, Delahunt E, Ekstrand J, Griffin D, Khan KM, Lovell G, Meyers WC, Muschaweck U, Orchard J, Paajanen H, Philippon M, Reboul G, Robinson P, Schache AG, Schilders E, Serner A, Silvers H, Thorborg K, Tyler T, Verrall G, Jan de Vos R, Vuckovic,Z, Hölmich P. Doha agreement meeting on terminology and definitions in groin pain in athletes. Br J Sports Med. 2015;49:768-774. 